\title{
Controversia en torno al uso de timerosal en vacunas: de la evidencia a la toma de decisiones
}

\author{
Controversy regarding the use of thimerosal in vaccines: \\ evidence for decision making
}

$\mathrm{U}$ n grupo de expertos convocado por la Academia de Medicina de Chile, a solicitud del Ministerio de Salud, ha presentado el informe titulado "Sintesis de evidencia sobre la seguridad de las vacunas que contienen timerosal". Recapitulando, en julio de 2010 inició su primer trámite constitucional en la Cámara de Diputados el proyecto de ley titulado "Elimina las vacunas multidosis con timerosal o compuestos organomercúricos" (boletín $\mathrm{N}^{\circ}$ 7036-11)1. Dicho proyecto de ley corresponde a una moción presentada por diputados de un amplio espectro político y se fundamentó en los siguientes argumentos: (i) la existencia de una posible asociación entre exposición a timerosal y trastornos del neurodesarrollo; (ii) la aplicación del "Principio Precautorio"; (iii) la presencia de timerosal en las vacunas reduciría la adherencia al programa de vacunación por parte de los usuarios y (iv) la presencia de timerosal en las vacunas potencialmente vulnera diversas garantías constitucionales (derecho a la protección de la salud, al acceso libre e igualitario a las acciones de promoción, protección, recuperación de la salud y de rehabilitación).

Entre los años 2010 y 2013 el proyecto de ley aprobó las sucesivas instancias legislativas requeridas para convertirse en una ley de la República; sin embargo, su aprobación experimentó un importante revés a comienzos del año 2014 con la emisión de un "veto supresivo" por parte del Presidente de la República de aquel entonces. El haber llegado a la instancia del veto, luego de tres años y medio de tramitación parlamentaria, fue la consecuencia de diversos errores cometidos durante el proceso, siendo el principal de ellos el no haber conformado desde un principio un mapa de actores válidos, en el cual se incluyese a representantes de las sociedades científicas, ya que éstas son depositarias del conocimiento científico-técnico y sus directivas son portavoces legítimos del conjunto de profesionales que le conforman. La incorporación de diversas sociedades científicas a la discusión -no por invitación, sino más bien como reacción frente a una ley ad portas de aprobarse- precipitó la emisión del veto presidencial, recurso que permitió detener el proceso legislativo, ofreciendo una valiosa oportunidad para enmendar la forma en que se conducía dicho proceso.

Luego de numerosas conversaciones entre partidarios y detractores del proyecto de ley (entre los últimos se encuentra la Sociedad Chilena de Infectología), las partes consensuaron y firmaron una declaración conjunta, cuyo tercer punto señala lo siguiente (cito textual):

"Los firmantes concordamos en la importancia de contar con un espacio de diálogo y consenso que recoja las inquietudes de los distintos actores de la sociedad y que se base en el análisis crítico de la evidencia científica disponible para tomar decisiones que eventualmente puedan modificar el plan nacional de inmunizaciones".

El informe presentado por el grupo de expertos convocados por la Academia Chilena de Medicina materializa lo señalado en el párrafo anterior. Dicho informe corresponde a una revisión sistemática de la literatura -el más alto nivel de evidencia-, cuyo objetivo fue (cito textual) "Evaluar si el timerosal contenido en las vacunas constituye un factor de riesgo de trastornos de neurodesarrollo en niños". Para ello, los autores utilizaron las revisiones sistemáticas ya existentes (cinco en total) como fuente de estudios primarios, complementadas con los estudios primarios publicados con posterioridad a éstas. En total fueron identificados y seleccionados 32 estudios, 27 provenientes de las revisiones sistemáticas ya existentes y cinco provenientes de la búsqueda adicional de estudios primarios en bases de datos electrónicas (Medline, CENTRAL, EMBASE, LILACS y WHO ICTRP). El análisis crítico de la evidencia permitió a los autores plantear las siguientes conclusiones (cito textual):

- La evidencia acumulada muestra que no existe asociación entre timerosal y autismo.

- La certeza de la evidencia es moderada**, principalmente por provenir de estudios con alto riesgo de sesgo.

\footnotetext{
* "Principio Precautorio". El enfoque "mejor seguro que lamentar" para evaluar y manejar riesgos en salud, especialmente aquellos asociados con riesgos medioambientales. Donde hay evidencia suficiente para creer que un riesgo existe, la prudencia, normas éticas y valores requieren que se tomen acciones para reducir o eliminar dicho riesgo, aún si la evidencia no es concluyente.
} 
- Nuestras conclusiones son concordantes con las conclusiones de las revisiones sistemáticas individuales identificadas, en especial con aquellas más recientes, de mayor calidad y que han logrado estimar un resultado acumulado mediante meta-análisis.

- Nuestros resultados son concordantes con las recomendaciones emitidas por la Organización Mundial de la Salud.

- La única forma de obtener una mayor certeza sería mediante la realización de un estudio controlado, de asignación aleatoria, o algún otro diseño con menor riesgo de sesgo, como por ejemplo una serie de tiempo interrumpida, que podría ser más factible de realizar en el contexto actual.

La evidencia científica ha sido presentada por el grupo de expertos, y corresponde los interesados evaluarla críticamente. Surge entonces la siguiente pregunta: ¿cuáles son los pasos a seguir? Un primer paso sería evaluar la realización de un nuevo estudio que permita elevar el nivel de certeza de la evidencia (estudio controlado, de asignación aleatoria, serie de tiempo interrumpida), teniendo claro las implicancias de esta decisión (por ejemplo, ¿quién(es) realizaría(n) el estudio? ¿cuánto tiempo estamos dispuestos a esperar sus resultados?). Cuando demos por cerrada la etapa de generación y/o búsqueda, síntesis y análisis de la evidencia disponible transitaremos hacia una nueva etapa, denominada "de la evidencia a la decisión". Un concepto fundamental de esta etapa sostiene que, para la toma de decisiones, "la evidencia es necesaria, pero no es suficiente". Ello implica que la toma de decisiones no debe basarse únicamente en la evidencia científica y en su certeza, sino también exige considerar un conjunto de factores claves. Para ejemplificar lo anteriormente señalado, si reducimos el problema a la decisión de cambiar desde una formulación de vacuna con timerosal (por ejemplo, vacuna pentavalente) hacia una formulación de vacuna sin timerosal (por ejemplo, vacuna hexavalente) por las razones aludidas en el proyecto de ley, debiesen considerarse al menos los siguientes elementos ${ }^{3,4}$ :

- Determinación si el problema en cuestión (trastornos del neurodesarrollo en niños) es una prioridad de salud para Chile.

- Magnitud del problema (es decir, el número de potenciales afectados por trastornos del neurodesarrollo atribuibles al uso de vacunas con timerosal).
- Beneficios y daños secundarios al cambio, y su adecuado balance (por ejemplo, ¿es la efectividad del componente anti-pertussis de la vacuna hexavelente igual de efectivo que el de la vacuna pentavalente? ¿Producen ambas vacunas el mismo nivel de inmunidad de rebaño?).

- Costo-efectividad de la vacuna hexavalente respecto a la vacuna pentavalente, costos económicos del cambio para el Programa Nacional de Inmunizaciones, y costo de oportunidad para el país (ante recursos limitados, asignarlos para implementar una medida implica dejar de asignarlos para implementar otra).

- Factibilidad de implementar el cambio (¿requiere la distribución, almacenamiento y administración de la vacuna hexavalente alguna logística especial?).

- Disponibilidad de la vacuna hexavalente (¿existe el riesgo de que nuestra demanda de vacuna hexavalente no sea cubierta?).

- Aceptabilidad social del cambio (¿cómo reaccionarán los padres y/o madres de los niños al cambio de vacuna pentavalente por hexavalente?).

- Seguridad de las nuevas formulaciones (¿cómo es el perfil de reactogenicidad de la vacuna hexavelente en comparación a la vacuna pentavalente?).

- Consideraciones especiales respecto a subgrupos de personas (¿existen subgrupos de personas que, por ejemplo, no puedan recibir vacuna hexavalente?).

- Aspectos sociales (¿el cambio de formulación de vacuna afecta la equidad en salud?).

- Aspectos legales (¿qué requisitos legales se requieren para implementar el cambio?).

- Aspectos éticos (¿existen otras prioridades en salud que se vean afectadas por el cambio de vacuna pentavalente por hexavalente?).

Para conducir adecuadamente el tránsito desde la evidencia a la decisión, considero necesario contar con algún marco teórico (por ejemplo, Evidence to Decision Frameworks, Evaluación de Tecnologías Sanitarias, ETESA $\left.^{* * *}\right)^{3,5}$ y apoyo de expertos ad hoc que nos permita sistematizar y transparentar la toma de decisiones, asegurar que los factores importantes que determinan la decisión sean tomados en cuenta, y apoyarnos en la resolución de controversias. De este modo, podremos informar a quienes finalmente deben tomar la decisión acerca de los elementos a favor y en contra de cada opción, con su

** El sistema GRADE reconoce cuatro niveles de certeza de la evidencia: Alto, Moderado, Bajo y Muy Bajo. La definición de "Moderado" es la siguiente: "El efecto real de la intervención probablemente se encuentra cerca de nuestra estimación, pero existe la posibilidad de que sea sustancialmente diferente"2.

*** La evaluación de tecnologías sanitarias (ETESA) corresponde a la investigación que examina las consecuencias técnicas, sociales, económicas, éticas y legales derivadas del uso de las tecnologías (o intervenciones) sanitarias, que se producen a corto y largo plazo y que pueden ser tanto directas como indirectas y tanto sobre los efectos deseados como sobre los indeseados . $^{5}$ 
respectiva justificación. Posteriormente, una vez que se ha tomado la decisión, es necesario planificar la forma en que ésta será comunicada a la sociedad civil y en que será implementada a lo largo del país. Por último, para cerrar el proceso exitosamente, deberíamos monitorizar los efectos de la decisión, así como evaluar periódicamente su idoneidad.

La forma originalmente planteada para dar solución a la controversia - prohibir el uso de timerosal en las vacunas a través de una ley- está muy lejos de ser la forma idónea para tomar decisiones en materias de salud, especialmente cuando éstas tienen un fuerte componente técnico. Tenemos la valiosa oportunidad de resolver esta controversia de manera ordenada, participativa y transparente, marcando un precedente frente a situaciones similares que se presenten en el futuro.

Jaime Cerda Facultad de Medicina, Pontificia Universidad Católica de Chile. Integrante Comité Consultivo de Inmunizaciones, Sociedad Chilena de Infectología.
Conflictos de interés: Ninguno relacionado al tema expuesto.

Correspondencia a: Jaime Cerda Lorca jcerdal@gmail.com

\section{Referencias bibliográfícas}

1.- Proyecto de ley "Elimina las vacunas multidosis con timerosal o compuestos organomercúricos" (boletín $\mathrm{N}^{\circ}$ 7036-11) Disponible en www.diputados.cl (Accedido el 28 de julio de 2015).

2.- Neumann I, Pantoja T, Peñaloza B, Cifuentes L, Rada G. El sistema GRADE: un cambio en la forma de evaluar la calidad de la evidencia y la fuerza de las recomendaciones. Rev Med Chile 2014; 142: 630-5.

3.- GRADE/DECIDE. Interactive Evidence to Decision Framework (iEtD). Disponible en: http://ietd.epistemonikos. org (Accedido el 28 de julio de 2015).

4.- World Health Organization (2015). Guidance forthe development of evidence-based vaccine related recommendations. Version 4. Disponible en: http://www. who.int/immunization/sage/Guidelines_development recommendations.pdf (Accedido el 28 de julio de 2015).

5.- Castillo-Riquelme M, Espinoza M. Evaluación de tecnologías sanitarias. Rev Med Chile 2014; 142 (Suppl 1): 4-5. 